\title{
Cardiac Care for Patients with Down's Syndrome: The Great Divide
}

\author{
Kristina Paulus ${ }^{1}$, Osama El Shazali ${ }^{2}$, Hamed Ahmed ${ }^{2}$, Seham Ahmed ${ }^{2}$, Hafiz El-Shazali², Markus Khalil ${ }^{1}$, \\ Gerardus Bennink ${ }^{1}$, Raman Krishna Kumar ${ }^{3}$ and Narayanswami Sreeram ${ }^{1 *}$
}

${ }^{1}$ Heart Center, University Hospital of Cologne, Germany

${ }^{2}$ Department of pediatrics, University of Khartoum, Sudan

${ }^{3}$ Amrita Institute of Medical Sciences, India

Submission: June 05, 2017; Published: August 10, 2017

*Corresponding author: Narayanswami Sreeram, Heart Center, University Hospital of Cologne, Germany, Tel: +49 221-478-32517; Fax: +49 221-

478-32515; Email: n.sreeram@uni-koeln.de

Abstract

Background: There are great discrepancies in the quality and level of care offered to patients with Down's syndrome and a structural heart defect. While this is well recognized, there are few comparative data in the current era.

Aim: To compare the level of care offered, and the outcome of three cohorts of children with Down's syndrome and congenital heart disease, from three tertiary referral centers.

Methods: A continuous case series of patients referred to each of the tertiary referral centers was followed up to evaluate the treatment and outcome.

Results: Early diagnosis and routine early repair of the heart defect with good outcome is the norm in the developed world. This contrasts with late presentation with congestive heart failure and failure to thrive, lack of early and appropriate therapy, a high rate of loss of followup, and a low rate of timely correction in the developing world. Given appropriate resources and training, excellent surgical results can be obtained universally.

Conclusion: While the majority of children with Down's syndrome and a structural heart defect can be adequately treated to ensure long term survival and a good quality of life, such therapeutic options are not universally available or applied.

Keywords: Down's syndrome; Heart disease; Quality of care

\section{Introduction}

Down's syndrome is the commonestchromosomal abnormality in man. The risk of a congenital heart defect in children with Down's syndrome is around $50 \%$. The most common associated defect is the atrioventricular septal defect, although differences in the frequency of certain types of defects may occur depending on the geographical location [1]. In the developed world, medical care for infants and children with Down's syndrome has improved tremendously in the last four decades, resulting in a better quality of life and higher life expectancy. One of the major factors for this improvement has been the prompt diagnosis and management of the associated cardiac defects, which if untreated may lead to a variety of immediate and late complications such as failure to thrive, recurrent infections, congestive heart failure, and eventually to the development of the Eisenmenger syndrome in some patients. The median age of death for patients with Down's syndrome and an associated cardiac defect in the United States has increased in the last years. In 1997 it was 49 years compared to 25 years in 1983. Few data are available regarding organized medical care for children with Down syndrome in the developing world. The mortality rate in the first years of life may be postulated to be higher due to later diagnosis and the lack of facilities for appropriate and timely interventions for these children [1-3]. The aim of this study was to compare the outcome of the medical care of infants with Down's syndrome and a congenital heart defect from three centers, representing the developing world and Western Europe. 


\section{Materials and Methods}

These are the results of a cross-sectional descriptive study which was done in all the neonatal units and children's hospitals in Khartoum, Sudan and of a retrospective study of infants and children with Down's syndrome and a congenital heart defect seen at the cardiology departments of Amrita Institute of Medical Sciences, Kochi, India, and of the University Hospital of Cologne.

\section{Patient inclusion}

All infants seen in all the Khartoum (Sudan) state neonatal units and children's hospitals with a clinical diagnosis of Down syndrome during a six month study period starting from September 2011 were prospectively included in the study $(\mathrm{n}=109)$. Only patients less than 12 months of age were included. Infants were divided into three groups based on age: group 1 aged less than a month $(n=41)$, group 2 with an age between one and six months $(n=37)$ and group 3 with an age older than six months $(n=31)$. Failure to thrive was defined as a weight for age which falls below the $5^{\text {th }}$ percentile. Informed consent was obtained from all the parents. A pre-tested questionnaire was filled and all children underwent a detailed clinical examination and echocardiography. All the echocardiographic examinations were done by one person using GE vivid 5 (GE Waukesha, WI). For the purpose of the study only hemodynamically significant lesions were included (a patent oval foramen or a clinically insignificant patent duct were excluded).

From the database of the Amrita Institute of Medical Sciences (India) 418 consecutive patients with Down's syndrome were identified between November 2005 and April 2012. Of these, a structural cardiac defect was present in 256 patients (63.4\%). The defect was identified at $<1$ month of age in 124 patients (group 1 ), at between 1 and 6 months in 113 (group 2), and at later than 6 months in the remainder (group 3). All defects were confirmed by routine transthoracic echocardiography.

From the database of the university hospital of Cologne (Germany) 92 consecutive infants with Down syndrome and an associated significant congenital heart defect, identified and managed between January 2003 and December 2014 were included. All patients belonged to group 1 (diagnosis prior to 1 month of age). Parameters that were evaluated included prenatal knowledge of Down syndrome, prenatal diagnosis of a heart defect, age at the time of surgery, and outcome of surgery. In all patients, the diagnosis was always confirmed by prenatal or postnatal chromosomal analysis.

\section{Results}

\section{Khartoum}

Of the three groups 47 infants (43.1\%) had a congenital heart defect. The most common defect was the atrioventricular septal defect (AVSD; 32\%) followed by an isolated VSD, secundum atrial septal defect (ASD), and persistently patent arterial duct (PDA).
All of these defects were single lesions. Approximately $57 \%$ of all the infants with Down's syndrome, independent of whether they had a congenital heart defect, were undernourished and only $1 \%$ was overweight. Of the 47 infants with an associated heart defect, $41(87 \%)$ were failing to thrive. None of the patients was offered palliative or corrective surgery at the time of initial diagnosis, due to lack of adequate facilities and funds for diagnostic cardiac catheterization or open heart surgery.

\section{Kochi}

The distribution of defects in this cohort was as follows: isolated VSD in 72 patients; AVSD in 70 patients; PDA in 43 patients; ASD in 32 patients, tetralogy of Fallot in 10 patients, Ebstein's anomaly in 4 patients. In addition 25 patients had combined lesions (VSD+PDA in 14, ASD+PDA in 6, AVSD+PDA in 5). Corrective surgery was performed in 104 patients (VSD=37; $\mathrm{AVSD}=25 ; \mathrm{PDA}=14 ; \mathrm{ASD}=4$; Ebstein's anomaly=3, tetralogy of Fallot=5, combined lesions $=16$ ). The age at corrective surgery was $<6$ months in 27, between 7 and 12 months in 45, 13 to 23 months in 20 , and at $>24$ months in 12 patients respectively. A single surgical procedure was performed in 100 of the 104 patients. There were no surgery related deaths. None of the patients has been lost to follow-up. Patients who did not receive surgical correction fell into one of two categories: those with minor defects not requiring surgery $(n=58)$, and those who refused surgery, chiefly for financial reasons $(\mathrm{n}=94)$.

\section{Cologne}

The medical records of the department of pediatric cardiology at the University hospital of Cologne were reviewed to identify the treatment strategies and outcome of a consecutive cohort of patients with Down's syndrome between 2003 and 2014. Of 209 patients confirmed to have Down's syndrome, a cardiac anomaly was present in 102 infants (48.8\%). Thirty eight of these were identified prenatally to have Down's syndrome (all confirmed by chromosomal analysis), and in all of them the structural cardiac lesion was also correctly diagnosed before birth. In the remaining patients, the suspicion of a Down's syndrome was raised immediately after birth, and confirmed by chromosomal analysis. All diagnoses (clinical diagnosis of Down's syndrome and the cardiac lesion) were established within the first week of life. Postnatal cardiac diagnoses requiring further management included AVSD ( $n=56)$, AVSD with tetralogy of Fallot $(n=5)$, isolated VSD ( $n=17)$, coarctation of the aorta $(n=3)$, secundum ASD $(\mathrm{n}=2)$, a hemodynamically significant PDA $(\mathrm{n}=2)$. In 17 infants a minor defect was identified which did not require further medical management (persistent patency of the duct, a small shunt at atrial level, a hemodynamically insignificant VSD).

Forty eight infants were operated within the first 6 months of life; 19 infants underwent surgery at between 7 and 12 months of age, 5 children at between 13 and 23 months of age, and 7 children at beyond 24 months of age. Fifteen children had more than one surgical procedure (PA banding $n=12$, re-repair of the 
left atrioventricular valve following initial complete repair of AVSD $n=1$, late replacement of the left AV valve with a mechanical prosthesis following AVSD repair $n=1$, closure of a significant residual VSD $n=1)$. Six children underwent a transcatheter therapeutic procedure (ASD closure in 2, PDA closure in 4).

\section{Follow-up}

Khartoum: As of December 2016, 86 of the 109 Sudanese patients have been routinely followed up, while 23 patients have been lost to follow-up, due to non-attendance. Of the 86 followed up patients, 21 have undergone surgery. This included complete repair of AVSD outside the Sudan ( $\mathrm{n}=8$, with 2 early deaths), 7 children who underwent pulmonary artery banding for AVSD, 3 children who underwent PA banding for VSD, and 3 patients who underwent late repair of an isolated VSD in the Sudan. In addition to the two surgery-related deaths, five additional children have died from a combination of complications of heart disease, failure to thrive and infection.

Kochi: All patients with and without surgical repair have undergone routine follow-up. None of the surgical group has required a second procedure, and none of them is on long-term medications. Among patients who refused surgery, 14 have died from complications including recurrent infections and Eisenmenger reaction.

Cologne: 1 patient died during the early post-operative period due to intractable septicemia associated with multi-organ failure. Seven patients needed a permanent pacemaker at follow-up; two due to complete AV block after repair of AVSD, and five patients due to late onset of sinus node disease $(n=2)$, or intermittent $A V$ block at follow-up $(n=3)$. Within 3 months of surgery, 77 of the 85 patients who had undergone surgery or transcatheter therapy were not taking any cardiac medication, with the exception of coumadin or aspirin.

\section{Discussion}

The purpose of this study was to evaluate the available data of the outcome of children with Down syndrome and a congenital heart defect and to describe the wide discrepancies in availability of care between different centers and countries. Congestive cardiac failure is the commonest cause of death in the first 6 months of life in infants with a structural cardiac defect, and cardiac disease accounts for most deaths in children with Down's syndrome in the first two years of life [4-6]. While life expectancy and quality of life has improved during the last years in the western world, very limited data exist concerning the management of these children in developing countries. In 1981 an editorial in the British Medical Journal stated that "if the child is one of the one quarter of newborns with Down's syndrome with congenital defect of the heart or other organs, then treatment may reasonably be withheld" [7]. In concordance with the poor surgical outcomes in some units in the developed world, this argument was further supported from the data from the Brompton hospital in the UK [8]. The authors stated that "remembering the mortality figures for a surgical protocol must include preoperative mortality, perioperative mortality, and late mortality, few units, including our own, will be able to recommend operation on the grounds of improved survival through childhood" [8]. They further suggested that a different (conservative) approach should be taken towards the management of complete AVSD in patients with Down's syndrome as opposed to those with no chromosomal abnormality. This illustrates that medical and surgical treatment of children with Down's syndrome and CHD was not always universally agreed upon, and current practice in the developed world reflects a gradual change in attitudes towards ensuring a good quality of life and increasing life expectancy by correcting the cardiac defect.

The data from Kochi confirm that excellent surgical outcomes can be obtained even in a limited resource setting. Cardiac surgery however has a major micro-economic impact. In a recent study from Kochi it was shown that median expense for hospital admission and cardiac surgery was 0.93 times the annual family income of affected patients [9]. Surgical admission also resulted in a median loss of 35 man- days and 15 job-days respectively, with one in two families reporting overwhelming to high financial stress related to surgery. Significantly around $50 \%$ of the families also had to borrow money during follow-up after surgery, to cover the costs [9]. These retrospective data also show a wide variation in the prevalence of congenital heart disease, and in the distribution of lesions depending on geographic location. These may reflect true racial or ethnic differences, although a referral bias cannot be excluded. In the developed world, given the establishment of routine prenatal and early postnatal screening, the diagnosis of Down's syndrome and coexisting cardiac disease can usually be established early, allowing appropriate care to be instituted immediately. Presentation with failure to thrive, or with other complications of congestive cardiac failure is therefore extremely uncommon. Similarly, the prevalence of Eisenmenger syndrome due to late detection or lack of facilities for timely surgical repair has also decreased in the current era.

In contrast and as has been highlighted in a recent editorial, pediatric cardiac surgical care is resource intensive, and only a fraction of patients in developing countries can expect to receive comprehensive care [10]. It is a paradox that with falling birth rates, access to prenatal diagnosis and possible termination of pregnancy, opportunities for optimal management are unequally distributed, favoring countries with higher incomes, a comprehensive, state sponsored health insurance system, and lower patient loads.

\section{References}

1. Vida VL, Barnoya J, Larrazabal LA, Gaitan G, de Maria Garcia F, et al. (2005) Congenital cardiac disease in children with Down's syndrome in Guatemala. Cardiol Young 15(3): 286-290.

2. Christianson AL (1996) Down syndrome in sub-Saharan Africa. J Med Genet 33(2): 89-92.

3. Tomlinson TW, Scott CH, Trotman HL (2010) Congenital cardiovascular lesions in children with trisomy 21 at the Bustamante hospital for children. Cardiol Young 20(3): 327-331. 
4. Tubman TR, Shields MD, Craig BG, Mulholland HC, Nevin NC (1991) Congenital heart disease in Down's syndrome: two year prospective early screening study. BMJ 302(6790): 1425-1427.

5. Laursen HB (1976) Congenital heart disease in Down's syndrome. Br Heart J 38(1): 32-38

6. Clapp S, Perry BL, Farooki ZQ Jackson WL, Karpawich PP, et al. (1990) Down's syndrome, complete atrioventricular canal, and pulmonary vascular obstructive disease. J Thorac Cardiovasc Surg 100(1): 115121

7. Cook RC, Evans RC, Goodall J, Sanders RK (1981) Paediatricians and the law. Br Med J (Clin Res Ed) 283(6305): 1543.
8. Bull C, Rigby M, Shinebourne EA (1985) Should management of complete atrioventricular canal defect be influenced by coexisting Down syndrome? Lancet 325: 1147-1149.

9. Raj M, Paul M, Sudhakar A, Varghese AP, Haridas AC, et al. (2015) Microeconomic impact of congenital heart surgery: results of a prospective study from a limited-resource setting. PLoS One 10(6): e0131348.

10. Kumar RK (2015) Universal heart coverage for children with heart disease in India. Ann Pediatr Cardiol 8(3): 177-183.

\section{Your next submission with Juniper Publishers} will reach you the below assets

- Quality Editorial service

- Swift Peer Review

- Reprints availability

- E-prints Service

- Manuscript Podcast for convenient understanding

- Global attainment for your research

- Manuscript accessibility in different formats ( Pdf, E-pub, Full Text, Audio)

- Unceasing customer service

Track the below URL for one-step submission https://juniperpublishers.com/online-submission.php 